\title{
WARMA: APLICATIVO MÓVIL PARA EL APRENDIZAJE DE MATEMÁTICAS Y LENGUAJE EN INSTITUCIONES EDUCATIVAS DE LAS COMUNIDADES QUECHUA HABLANTES EN AYACUCHO
}

\author{
WARMA: MOBILE APPLICATION FOR THE LEARNING OF \\ MATHEMATICS AND LANGUAGE IN EDUCATIONAL INSTITUTIONS \\ OF THE QUECHUA-SPEAKING COMMUNITIES IN AYACUCHO
}
Edgar Gutiérrez Gómez ${ }^{*}$, (D) Isaac Ocampo Yahuarcani², (D) Kay Dennise Jeri Lagos²,
Lelis Antony Saravia Llaja ${ }^{2}$

eggutierrez@unah.edu.pe; iocampo@udaff.edu.pe; kjeri@udaff.edu.pe; saravia.lelis95@gmail.com
1Universidad Nacional Autónoma de Huanta, Ayacucho, Perú
2Universidad de Ayacucho Federico Froebel, Ayacucho, Perú

*Correspondencia: Edgar Gutiérrez Gómez. Email: eggutierrez@unah.edu.pe

Recibido: 11.04.19 | Aprobado: 15.06.19

\section{RESUMEN}

Desde la Universidad de Ayacucho Federico Froebel (UDAFF) se viene impulsando un proyecto de investigación que busca la mejora de la enseñanza y aprendizaje de lenguaje y matemáticas para niños de educación inicial de poblaciones quechua hablantes de Ayacucho, a partir del uso de tecnologías móviles (teléfonos celulares y tabletas). Entre los resultados que se esperan de este proyecto es que los niños de educación inicial cuenten con instrumentos educativos digitales (desde teléfonos celulares y tabletas) con contenido adaptado a la realidad ayacuchana, y que favorezcan el aprendizaje de lenguaje y matemáticas en la lengua materna de sus padres (quechua hablantes), siendo accesible desde cualquier comunidad quechua hablante. Esto además servirá para la reducción de debilitamiento de la lengua materna quechua, así como la identidad cultural de las poblaciones de los pueblos andinos.

Palabras clave: Educación, Comunidades indígenas, quechua hablantes, tecnologías móviles, Chuschi.

\begin{abstract}
From the University of Ayacucho Federico Froebel (UDAFF) has been promoting a research project that seeks to improve the teaching and learning of language and mathematics for children in early education quechua speakers of Ayacucho, from the use of mobile technologies (cell phones and tablets). Among the results that are expected from this project is that the children of initial education have digital educational tools (from cell phones and tablets) with content adapted to the reality of Ayacucho, and that favor the learning of language and mathematics in the mother tongue of their parents (quechua speakers), being accessible from any quechua speaking community. This will also serve to reduce the weakening of the quechua mother tongue, as well as the cultural identity of the populations of the Andean peoples.
\end{abstract}

Keywords: Education, indigenous communities, quechua speakers, mobile technologies, Chuschi. 


\section{INTRODUCCIÓN}

La pérdida de las lenguas originarias y culturas locales forman parte de las problemáticas que enfrentan la mayoría de los pueblos andinos ayacuchanos. Aunque estos también constituyen graves problemas de índole nacional, latinoamericana e incluso mundial. El Perú es un país multicultural y multilingüista, sin embargo, estas fortalezas son poco valoradas por las instituciones nacionales en un país dominado por mayorías hispanohablante, son muy escasos los servicios públicos disponibles para poblaciones que usan lenguas originarias.

A nivel mundial existen más de 7000 lenguas, más del 40\% de estas posee problemas de vulnerabilidad o desaparición. "De los casi 7.000 idiomas existentes, la mayoría han sido creados y son hablados por pueblos indígenas que representan la mayor parte de la diversidad cultural del mundo" (UNESCO, 2019). Según, Moreno (2017) reporta:

En 2016 existían 7.097 lenguas vivas habladas en todo el mundo, según la página web Ethnologue. De estas, un tercio están en peligro de desaparecer. Como muestra esta infografía de Statista, además, los diez países con más idiomas suman 4.318 registros diferentes, es decir, el 61\% del total.

En el caso del Perú, El Comercio (2018) informa: "En nuestro país existe un total de 48 lenguas originarias: 44 amazónicas y 4 andinas, las cuales están agrupadas en 19 familias lingüísticas y constituyen medios de comunicación de 55 pueblos indígenas u originarios". Según datos del Minedu (2019): "En la actualidad se cuenta con 40 lenguas con alfabetos oficializados, dos en proceso de normalización (nanti y asheninka) seis en proceso de documentación (iñapari, chamicuro, omagua, muniche, resígaro, taushiro). Información reportada en forma oficial. Complementa UNESCO (2019) presentando mapa de lenguas indígenas:

Estas lenguas se dividen en dos grupos: las que están en peligro de extinción, que son aquellas habladas mayoritariamente solo por adultos; y las que se encuentran seriamente en peligro, que son habladas sólo por núcleos familiares o personas dispersas y que ya no se transmiten a las nuevas generaciones.

Por otro lado, es válido destacar a otros países con diversidad lingüística como Brasil en Lenguas (2018) destacando: "En la actualidad, son habladas por los pueblos indígenas del Brasil más de 150 lenguas y dialectos". Por otra parte, está México: "El Instituto Nacional de Lenguas Indígenas indica que casi seis millones de mexicanos hablan una lengua indígena. Hablan 364 variantes lingüísticas asociadas a 68 lenguas, que a su vez pertenecen a 11 familias lingüísticas distintas" (Vida, 2018). En Bolivia existen similares características, según UMSA (2018): ... 30 lenguas nativas en Bolivia (de las 36 reconocidas por su Constitución) se encuentren en un nivel vulnerable o en peligro de extinción". En el territorio del "Ecuador se hablan 14 lenguas ancestrales" (Foros Ecuador, 2019). Por otra parte, El Universo (2018) informa:

En Ecuador existen catorce lenguas indígenas y todas tienen un grado de vulnerabilidad, incluso el kichwa -que es la más utilizada y que ha desplazado a otras ancestrales-. El director del Instituto de Idiomas, Ciencias y Saberes 
Ancestrales (Iicsa), Luis Males explicó a la agencia Andes que, si las nuevas generaciones no continúan usándola en su diario vivir, podrían desaparecer.

En el territorio chileno, Nacional (2019) informa: "Este 21 de febrero y todo el resto del año 2019 invitamos a reconocer, valorar y hablar las 6 lenguas indígenas vigentes hoy en Chile y también a las que están en reconstrucción". Así mismo es pertinente mencionar que a nivel mundial cientos de lenguas han desaparecido y decenas de ellas que cuentan con pocos hablantes, también están en camino a la desaparición. Según informes que: "Cada dos semanas muere una lengua. Es probable que a finales de siglo hayan desaparecido casi la mitad de las cerca de 7.000 lenguas que se hablan hoy en el mundo" (National Geographic, 2018). En el caso del Perú podemos destacar a las lenguas Taushiro, Resígaro e Ikitu en Loreto, las tres cuentan con escasos número de hablantes, sobre todo las primeras dos, que según el Ministerio de Cultura que apenas disponen de un hablante (Minedu, p,68).

Entre los principales factores asociados al debilitamiento y desaparición de las lenguas originarias en el Perú se encuentran una serie de acciones reduccionistas impulsadas desde que el país era colonia española, no se sabe a ciencia cierta cuantas lenguas se hablaban, lo que si se conoce es que hasta la iglesia impulsó la difusión y homogenización de la lengua indígena basada en el Quechua, de allí posiblemente surgen tantos variantes del Quechua que existen en el Perú, entre los que podemos mencionar al Quechua chanca (Ayacucho), Quechua cuzqueño, Quechua cajamarquino, Quechua yauyino, Quechua ancashino, así mismo en la Amazonía tiene variantes como el Quechua del Napo, el Quechua del Pastaza, el Quechua de Lamas, a la vez algo parecido pasa con el Quechua en la mayoría de países vecinos del Perú, pues también se registran variantes del Quechua en Argentina, Brasil, Colombia, Ecuador, Bolivia, Chile, etc. "Además, el quechua adquirió una función importante en los niveles regionales y locales de la administración". (El quechua, s/f, p,3). En la práctica el quechua es la lengua más expandida y vigorosa de Sudamérica, en comparación de las otras más de 300 lenguas.

Hablar en quechua o en cualquiera de las otras 47 lenguas peruanas convierte a sus hablantes en poblaciones excluidas, la lengua se convierte en un factor de menosprecio social en el contexto de las ciudades, en un factor que incluso hace que las poblaciones se avergüencen de su cultura. Existen decenas de casos de miembros de pueblos originarios que han cambiado sus apellidos, que ocultan su lengua materna, que niegan el conocimiento de su cultura, siendo estos factores importantes para que diversas lenguas peruanas se encuentren en estado de vulnerables o desaparición.

Otro factor importante lo constituyen las débiles políticas y servicios públicos relacionados a las poblaciones originarias, las autoridades no priorizan servicios públicos fuera de las ciudades, dejando a un lado a pueblos y comunidades alejadas, y esto se agravan paulatinamente, si en estos pueblos no se habla español.

Otro factor vital lo constituye la calidad de los servicios educativos en comunidades que no hablan español. Y es que por parte del estado existen las denominadas instituciones educativas que utilizan un sistema denominado Educación Bilingüe Intercultural (EIB) impulsada por el Ministerio de Educación con Resolución Ministerial No 629-2016MINEDU, que si bien es cierto están extendidas por gran parte de departamentos del 
Perú, sin embargo, su implementación históricamente no ha alcanzado resultados que fortalezcan o revitalicen las lenguas y culturas originarias.

Para resarcir esta situación de los pueblos indígenas, víctimas de la invasión y dominación, el Gobierno estableció el Sistema de Educación Bilingüe de la Selva dirigido por el Instituto Lingüístico de Verano de Yarina Cocha-Pucallpa, que fue todo un roselitismo religioso. Por lo que, a partir de la Evaluación realizada en el periodo de la Reforma de la Educación, el Gobierno asumió la direccionalidad de la Educación Intercultural Bilingüe en el país. Pero no tuvo la primacía en su organización ni en la atención que requieren los pueblos indígenas. (Rengifo, 2016).

Este servicio posee una serie de limitaciones y deficiencias que no satisfacen o ha favorecido los procesos educativos de los estudiantes, así como la sobrevivencia de las lenguas y el fortalecimiento y preservación de las culturas. Según documentos consultados: "En las últimas décadas han comenzado a implementarse distintas experiencias en América Latina de universidades indígenas y de universidades interculturales”. (Espinosa, 2017, p,111). De la misma forma se analiza: “... en los Estados Unidos, el Instituto Lingüístico de Verano (ILV), institución evangélica, que se convirtió en el aliado más importante de los Estados latinoamericanos en la causa asimilacionista y en cuyo marco se desarrollaron estos incipientes procesos educativos bilingües". (López y Küper, 2000, p,30). En tantos años se ha avanzado muy poco, por lo que se amerita probar nuevos métodos, instrumentos y procesos acompañado de apropiados niveles de inversión. Incluso el año 2019 ha sido declarado "Año Internacional de las Lenguas Indígenas" por la UNESCO: "La Asamblea General de las Naciones Unidas proclamó el Año Internacional de las Lenguas Indígenas con la

UNESCO como coordinadora del mismo". (2019). Fundamento importante de buscar la promoción y protección de las lenguas para la mejora de la calidad de vida de los que hablan la lengua.

Existen muchos factores que limitan el cumplimiento de los objetivos de las escuelas EIB, siendo el principal relacionado a nivel de inversión por parte del estado (si la educación pública en las ciudades tiene problemas, la educación en las comunidades quechua hablantes tiene muchas más complicaciones), así como los procesos al interior del servicio educativo.

En este contexto se han identificado los siguientes problemas que afrontan las escuelas IEB en el Perú:

- Se caracterizan por ser instituciones educativas con escaso número de docentes, la mayoría son colegios unidocentes que a su vez enseñan a todos los grados (docentes multigrados).

- Designación inapropiada de docentes, ya que son frecuentes los casos en que las instituciones responsables del sector educación designan como docentes a personas que desconocen la lengua y el contexto cultural de la comunidad.

- Las Instituciones educativas de las comunidades quechua hablantes no cuentan con material educativo que cubra el contenido curricular básico (libros, y otros textos o 
ilustraciones adaptadas a la localidad). Así mismo existe reducida producción e incorporación de contenido cultural de cada pueblo.

- Lejanía o aislamiento de las comunidades ayacuchanas dificulta y encarece la distribución de los materiales educativos. En algunos casos el material educativo si existe, sin embargo, no llegan a las instituciones educativas.

- Débil supervisión del servicio educativo en las comunidades quechua hablantes.

- Inexistente empleo de instrumentos tecnológicos de apoyo a los procesos de aprendizaje en Quechua Chanca.

- Alto ausentismo escolar en instituciones educativas de comunidades andinas.

Así mismo, es importante resaltar que los servicios educativos de la EBI no incorporan el uso de las tecnologías de la información como instrumentos de apoyo para los procesos de enseñanza y aprendizaje a pesar del enorme potencial, y de los usos y resultados que se vienen logrando en todo el mundo. En este contexto la Universidad de Ayacucho Federico Froebel (UDAFF), viene impulsando un proyecto de investigación que busca generar herramientas informáticas para apoyar la enseñanza y aprendizaje de lenguaje y matemáticas en las poblaciones quechua hablantes de Ayacucho.

Se han identificado como distritos pilotos a Chuschi (provincia de Cangallo), Acosvinchos y Quinua (en la provincia de Huamanga), todas con importantes poblaciones quechua hablantes y comunidades emblemáticas en Ayacucho. Entre los criterios de selección se han tomado en cuenta la importancia histórica de comunidades como Chuschi con un rol resaltado durante el conflicto armado de los años ochenta en el Perú. Treinta años después, Chuschi forma parte de las comunidades quechua hablantes que transitan en el olvido, como casi todas las comunidades quechua hablantes del departamento de Ayacucho.

En nuestras visitas a estos 3 distritos se pudo comprobar que en las instituciones educativas se enseña como primera lengua el castellano y como segunda lengua el Quechua, teniendo pocos docentes y los contenidos curriculares y materiales no adaptados a la lengua Quechua, en términos resumidos podemos indicar que el contenido curricular que se imparte en las instituciones educativas visitadas favorecen a que la lengua castellana se imponga sobre el Quechua, desvirtuando la esencia y los fines de impulsar educación bilingüe. Este problema es común en Ayacucho y en el resto del Perú.

Finalmente se ha construido un aplicativo informático prototipo para celulares que permitirá que los niños de educación inicial aprendan los elementos básicos de lenguaje y matemáticas en lengua quechua, seguidamente presentamos la metodología de construcción.

\section{METODOLOGÍA}

Consideraciones principales: Para el desarrollo de esta aplicación se tuvieron en cuenta aspectos importantes como la experiencia del usuario con el uso de tecnologías móviles (tabletas, Smartphone, laptops), su nivel de alfabetización, la disponibilidad de acceso a internet para la descarga de contenidos, entre otros. 
Es de mucha importancia mencionar que, a consecuencia del limitado acceso a internet en la zona de estudio, se optó desarrollar la aplicación para que el contenido que se muestre esté disponible de manera offline, el cual permite que la aplicación pueda ser expandible y masificable a otros dispositivos, dado que se puede transferir la aplicación y todo su contenido por otros medios a otros dispositivos móviles, ya sea a través de Bluetooth, wi-fi o dispositivos USB.

En el proceso de desarrollo de la aplicación se tuvo la participación de los miembros de la comunidad y docentes de las I.E. además de la colaboración de la Universidad Federico Froebel, desde la elaboración del contenido educativo de la aplicación hasta las pruebas de validación a las que se sometió la aplicación.

\section{Tabla 1}

Requisitos funcionales y no funcionales.

\begin{tabular}{|c|c|}
\hline Requisitos Funcionales & Requisitos No Funcionales \\
\hline $\begin{array}{l}\text { La aplicación debe funcionar de manera } \\
\text { OFF-LINE. } \\
\text { La navegación debe ser intuitiva, a traves de } \\
\text { botones e interfaces amigables para la fácil } \\
\text { navegación e interacción de un niño. } \\
\text { La aplicación debe estar organizada por } \\
\text { categorias de contenidos. } \\
\text { La aplicación debe mostrar contenido en } \\
\text { español y Quechua, siendo el sonido que se } \\
\text { reprodusca a traves de botones en la Lengua } \\
\text { Quechua. } \\
\text { El sonido que se reproduce en la aplicación } \\
\text { debe ser entendible y bien pronunciado. }\end{array}$ & $\begin{array}{l}\text { La aplicación debe ejecutarse en Dispositivos } \\
\text { de Diferentes medidas(Tablets, Smartphone, } \\
\text { etc), que dispongan de Sistema Operativo } \\
\text { Android. } \\
\text { El tamaño del paquete de la Aplicación no } \\
\text { debe ocupar mucho espacio de } \\
\text { almacenamiento en el Dispositivo (máximo } \\
20 \mathrm{MB} \text { ). } \\
\text { El contenido debe ser priorizado de acuerdo } \\
\text { a la curricula educativa de la Comunidad } \\
\text { Quechua hablante de Ayacucho. } \\
\text { Los contenidos del aplicativo deben ser } \\
\text { producidos con la participación de personas } \\
\text { Quechua hablantes de nacimiento, de } \\
\text { preferencia miembros de las comunidades. }\end{array}$ \\
\hline
\end{tabular}

\section{Proceso para el diseño de aplicaciones rurales}

\section{Recolección de requerimientos}

Una de las principales e importantes tareas que permitieron el desarrollo de la aplicación fue el poder identificar los requerimientos principales con los que contaría el software, para ello se realizaron visitas de campo hacia la comunidad de Chuschi, Quinua y Acosvinchos, el cual permitió tener un panorama más claro con respecto al producto que se debía desarrollar y el contenido que se debería mostrar dentro de la aplicación. Como primer aspecto nos interesaba conocer acerca del proceso de aprendizaje que se manejaba en la comunidad el cual pudimos tener acceso a través de pequeñas entrevistas a los pobladores y docentes de la IE, en el cual se les formulaba preguntas con respecto al contenido educativo básico con el que los niños empiezan a desenvolverse cognitivamente, el uso de dispositivos tecnológicos en actividades académicas y/o diarias (Tabletas, Smartphone, laptops), acceso al servicio de internet (conectividad), parte del contenido académico definido en su plan de enseñanza por parte de los docentes, entre otras preguntas que ayudaron a maquetar y tener noción de cómo debería estar estructurado la aplicación. 
Es así que se logró identificar categorías dentro de la aplicación, el cual están alineadas al contenido básico de asignaturas de matemáticas y Lenguaje, y podemos mencionar para la Categoría Matemáticas tenemos: Los Números, Los Números Ordinales, Figuras Geométricas, Los Colores, Signos Matemáticos, para la Categoría Lenguaje podemos mencionar: El Alfabeto, los valores, la familia, El cuerpo Humano, Los sentidos del cuerpo, La Fauna, Cosas y Expresiones.

\section{Producción de Contenidos para la App}

Por mencionar algunas actividades se trabajaron directamente con los docentes de las I.E. Bilingües, donde se pudo acceder a la curricula educativa de los grados de educación inicial y primaria, el cual fue importante a la hora de elaborar el contenido educativo que tendría la aplicación de acuerdo con el contenido descrito por cada curricula y a los indicadores que tendrían que satisfacer.

Como otro factor importante podemos mencionar la participación de los miembros de la comunidad, quienes definieron el contenido con respecto a la lista de animales, frutas, cosas y expresiones más comunes que se hablan en la comunidad, a partir de ello se pudo elaborar una ficha (ver Tabla 2 y 3 ) en el cual se listan los contenidos por cada categoría en el idioma español y dispone de columnas para su posterior traducción a la lengua materna de la comunidad.

\section{Tabla 2}

Alfabeto Quechua De Ayacucho

\begin{tabular}{lllll}
\hline \multicolumn{2}{l}{ Vocales Alfabeto Quechua } & \multicolumn{2}{l}{} \\
\hline \multicolumn{2}{c}{ A } & I & U \\
\hline \multicolumn{2}{l}{ Letras del Alfabeto Quechua } \\
\hline A & CH & H & I & K \\
L & LL & M & N & N \\
P & Q & R & S & T \\
W & Y & & & \\
\hline
\end{tabular}

Tabla 3

Formato de ficha de contenido de la aplicación.

\begin{tabular}{llll}
\hline $\mathbf{N}^{\circ}$ & Categoría & En español & Traducción Quechua \\
\hline 01 & Los Numeros & Uno & Huk \\
02 & Los Numeros & Dos & Iskay \\
03 & Los Numeros & Tres & Kimsa \\
04 & Los Numeros & Cuatro & Tawa \\
05 & Los Numeros & Cinco & Pichqa \\
06 & Los Numeros & Seis & Suqta \\
07 & Los Numeros & Siete & Qanchis \\
08 & Los Numeros & Ocho & Pusaq \\
09 & Los Numeros & Nueve & Isqun \\
10 & Los Numeros & Diez & Chunka \\
11 & Figuras Geométricas & Círculo & Ruyru \\
12 & Figuras Geométricas & Cuadrado & Tawa Kuchuyuq suytu \\
13 & Figuras Geométricas & Triangulo & Kimsa Kuchuyuq \\
14 & Figuras Geométricas & Grande & Machusu \\
\hline
\end{tabular}




\begin{tabular}{|c|c|c|c|}
\hline 15 & Figuras Geométricas & Mediano & Uchuy \\
\hline 16 & Figuras Geométricas & Pequeño & Uchuycha \\
\hline 17 & Signos Aritméticos & Más & Achka \\
\hline 18 & Signos Aritméticos & Menos & Rakisqa \\
\hline 19 & Signos Aritméticos & Resta & Qichuy \\
\hline 20 & Signos Aritméticos & Suma & Yapay \\
\hline 21 & Colores & Amarillo & Qillu \\
\hline 22 & Colores & Blanco & Yuraq \\
\hline 23 & Colores & Celeste & Cielu qina \\
\hline 24 & Colores & Marrón & Tumpa uqi \\
\hline 25 & Colores & Negro & Yana \\
\hline 26 & Colores & Rojo & Puka \\
\hline 27 & Colores & Verde & Quyu \\
\hline 28 & Colores & Gris & Uqi \\
\hline 29 & La Familia & Abuela & Paya \\
\hline 30 & La Familia & Abuelo & Taytalla \\
\hline 31 & La Familia & Papá & Tayta \\
\hline 32 & La Familia & Mamá & Mama \\
\hline 33 & La Familia & Hermano & Turi \\
\hline 34 & La Familia & Hermana & Ñaña \\
\hline 35 & La Familia & Amigo & Huayna \\
\hline 36 & La Familia & Amiga & Pisayki \\
\hline 37 & La Familia & Prima & Panicha \\
\hline 38 & La Familia & Primo & Huayqicha \\
\hline 39 & Los Valores & Amor & Kuyay \\
\hline 40 & Los Valores & Bondad & Chaninchay \\
\hline 41 & Los Valores & Generosidad & Allin runa \\
\hline 42 & Los Valores & Gratitud & Riksikuy \\
\hline 43 & Los Valores & Honestidad & Umalla kana \\
\hline 44 & Los Valores & Honradez & Ama suwa \\
\hline 45 & Los Valores & Humildad & Wacharuna \\
\hline 46 & Los Valores & Solidaridad & Yanapanakuy \\
\hline 47 & Los Valores & Sinceridad & Ama llulla \\
\hline 48 & Los Valores & Tolerancia & Suyanakuy \\
\hline 49 & Cuerpo Humano & Boca & Simi \\
\hline 50 & Cuerpo Humano & Brazo & Ricra \\
\hline 51 & Cuerpo Humano & Cabeza & Uma \\
\hline 52 & Cuerpo Humano & Dedo & Rawkana \\
\hline 53 & Cuerpo Humano & Mano & Maki \\
\hline 54 & Cuerpo Humano & Nariz & Sinqa \\
\hline 55 & Cuerpo Humano & Ojo & Nawi \\
\hline 56 & Cuerpo Humano & Pie & Chaki \\
\hline 57 & Fauna & Alpaca & Paqu \\
\hline 58 & Fauna & Búho & Tuku \\
\hline 59 & Fauna & Chancho & Kuchi \\
\hline 60 & Fauna & Cuy & Quwi \\
\hline 61 & Fauna & Gallina & Wallpa \\
\hline 62 & Fauna & Gato & Misi \\
\hline 63 & Fauna & Gavilán & Anka \\
\hline 64 & Fauna & Halcón & Waman \\
\hline 65 & Fauna & Hormiga & Sisi \\
\hline 66 & Fauna & Luciérnaga & Nina uru \\
\hline 67 & Fauna & Zorro & Atuq \\
\hline
\end{tabular}




\begin{tabular}{llll}
\hline 68 & Cosas & Catre & Kawitu \\
69 & Cosas & Cinturón & Chumpi \\
70 & Cosas & Cucharón & Wislla \\
71 & Cosas & Escoba & Pichana \\
72 & Cosas & Gorro & Chuku \\
73 & Cosas & Hacha & Qacha \\
74 & Cosas & Jarra & Chata \\
75 & Cosas & Leña & Yanta \\
76 & Cosas & Manta & Lliklla \\
77 & Expresiones & Bienvenido & Allin qamukuy \\
78 & Expresiones & Buenos días & Rimaycullayki - Allin punchaw \\
79 & Expresiones & Buenas tardes & Allin chinsinkuy \\
80 & Expresiones & Buenas noches & Allin tuta \\
81 & Expresiones & Adiós & Ripuchkani \\
82 & Expresiones & ¿Cómo te llamas? & ¿Imam sutiki? \\
83 & Expresiones & Mi nombre es (yo me llamo) & Sutiymi ... \\
\hline
\end{tabular}

Al término de la elaboración de la lista con el contenido de la aplicación identificado por categoría en la ficha de contenido, se realizó la traducción a la lengua materna correspondiente con apoyo de los miembros de la comunidad, donde cada palabra descrita en la ficha fue traducida y escrita por el mismo miembro de la comunidad en la lengua materna y con el alfabeto correspondiente a su lengua, obteniendo así una traducción confiable.

\section{Figura 1}

\section{Entrevista Con Docente De La Comunidad}

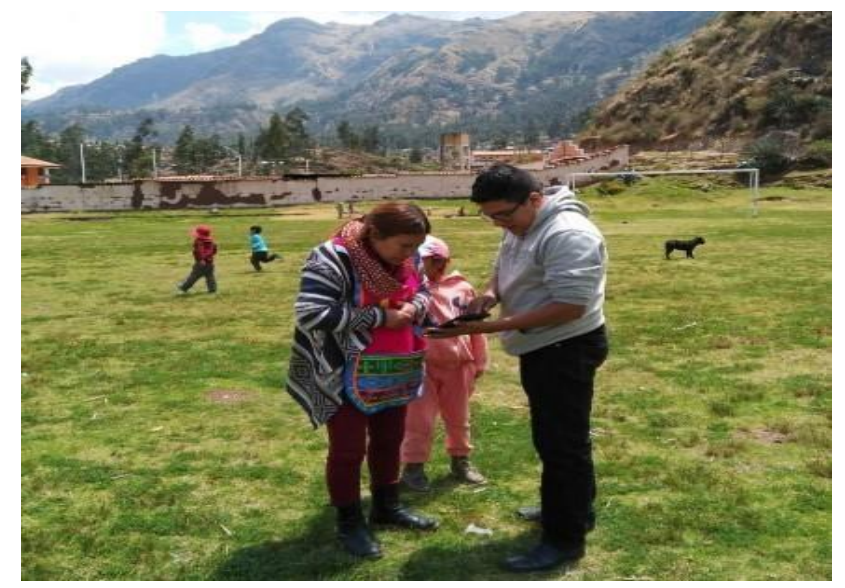

\section{Digitalización de Contenido}

Una vez identificado todos los requerimientos con los que contaría la aplicación y con la ficha de contenido totalmente completada se procedió a la grabación del sonido y pronunciación por cada palabra del contenido traducido, para ello se necesitó de un miembro de la comunidad que estuviera en la capacidad de pronunciar de forma fluida cada palabra traducida de la ficha de contenido en su lengua, para ello se llevó a cabo un consenso entre los hablantes de la lengua Quechua de la comunidad, donde se les preguntó quién de los miembros de la comunidad era la persona indicada para la realización de la labor de grabación de la pronunciación, teniendo en cuenta la consulta de los miembros más antiguos de la comunidad. Este proceso forma parte importante en el desarrollo de la aplicación dado que influirá mucho en cómo los usuarios finales puedan 
aprender el buen pronunciamiento de cada palabra en la lengua materna (acento, pausa, tiempo).

A partir de la ficha de contenido de la aplicación, con todas las palabras en español y traducido a la lengua materna, se elaboró una base de datos el cual contiene información de todo el contenido de la ficha y la voz pronunciada en la lengua por cada palabra, esta base de datos es utilizada en el desarrollo de la aplicación para que pueda ser utilizada de manera off-line por el usuario.

\section{Diseño de la Aplicación}

El diseño de la aplicación juega un papel muy importante en el desarrollo de la aplicación, ya que es en este proceso donde se define de qué manera estará distribuida toda la información y el contenido que se ha recolectado durante el proceso recolección de requerimientos y digitalización de contenido.

Para la elaboración del diseño de la aplicación se tuvo en cuenta la experiencia de los miembros de la comunidad respecto con el uso de dispositivos móviles (tabletas, Smartphone), la interacción con las aplicaciones que disponían, el número de clics que necesitaba para poder llegar a algún tipo de contenido que deseaba ver, y sobre todo la interface gráfica, tendríamos que desarrollar una interface gráfica donde el usuario no se sienta aburrida y toda la información y contenido estuviera al alcance en pocos clics de la aplicación, es decir, que la aplicación sea sencillo y con el contenido de fácil acceso, en la Fig. 2 podremos observar la distribución del contenido para el interfaz gráfica principal de la aplicación, donde el usuario tendrá a disposición a primera vista las categorías de la aplicación y en segunda vista el contenido por cada categoría.

\section{Validación y retroalimentación}

Hasta este paso la aplicación se encuentra en su fase casi final, este proceso de desarrollo de los prototipos de la aplicación, se realizan pequeñas reuniones con los miembros de la comunidad y con los docentes de la I.E. para que realicen la revisión correspondiente con respecto al contenido de la aplicación, es en este punto donde se hace las observaciones y recomendaciones por parte de los usuarios retroalimentando así la aplicación para llegar a una versión final de acuerdo a las necesidades de la comunidad.

\section{Figura 2}

\section{Boceto De La Distribución Del Contenido De La Aplicación}

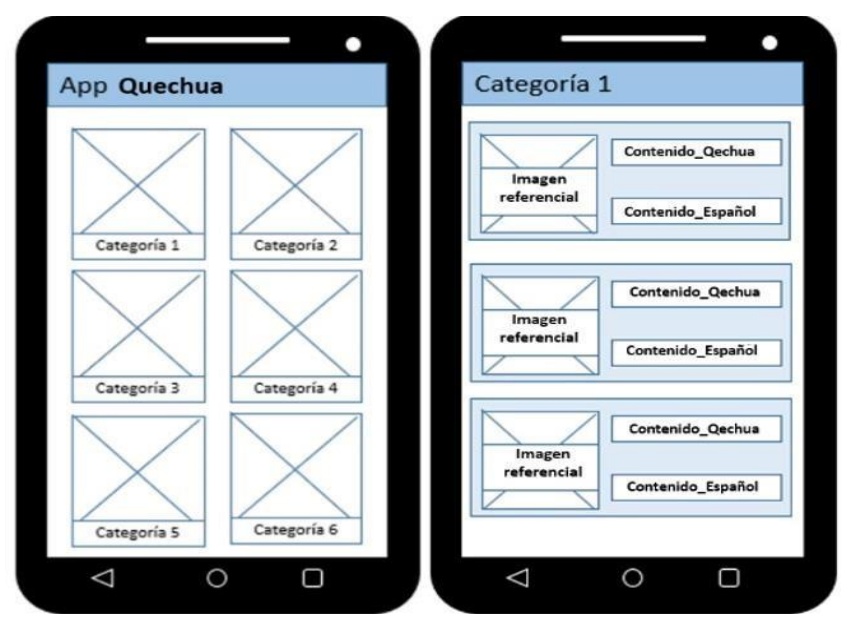




\section{Figura 3}

Interfaz, Beta De La Aplicación

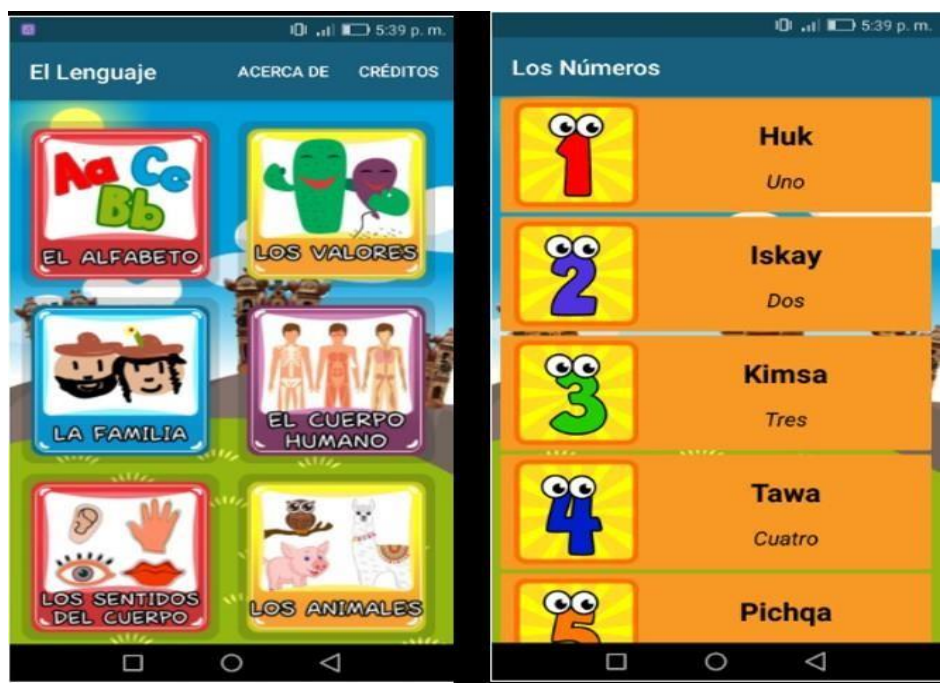

Finalmente, se realizaron pruebas de usabilidad donde, se aplicó la métrica subjetiva tal como se propone en el modelo mGQM (móvil Goal Question Metric) descrito por Enríquez J. y Casas S. en su artículo sobre usabilidad en aplicaciones móviles, ahí mencionan que el uso del marco de usabilidad considera 3 elementos principales: los elementos del círculo exterior conformado por cuatro factores (Usuario, Tecnología, Tarea/Actividad y Entorno), los elementos del círculo interior conformados por las dimensiones de usabilidad y la caja de la parte superior conformada por las consecuencias de usabilidad con respecto a la aplicación.

\section{Figura 4}

\section{Framework De Usabilidad Móvil}

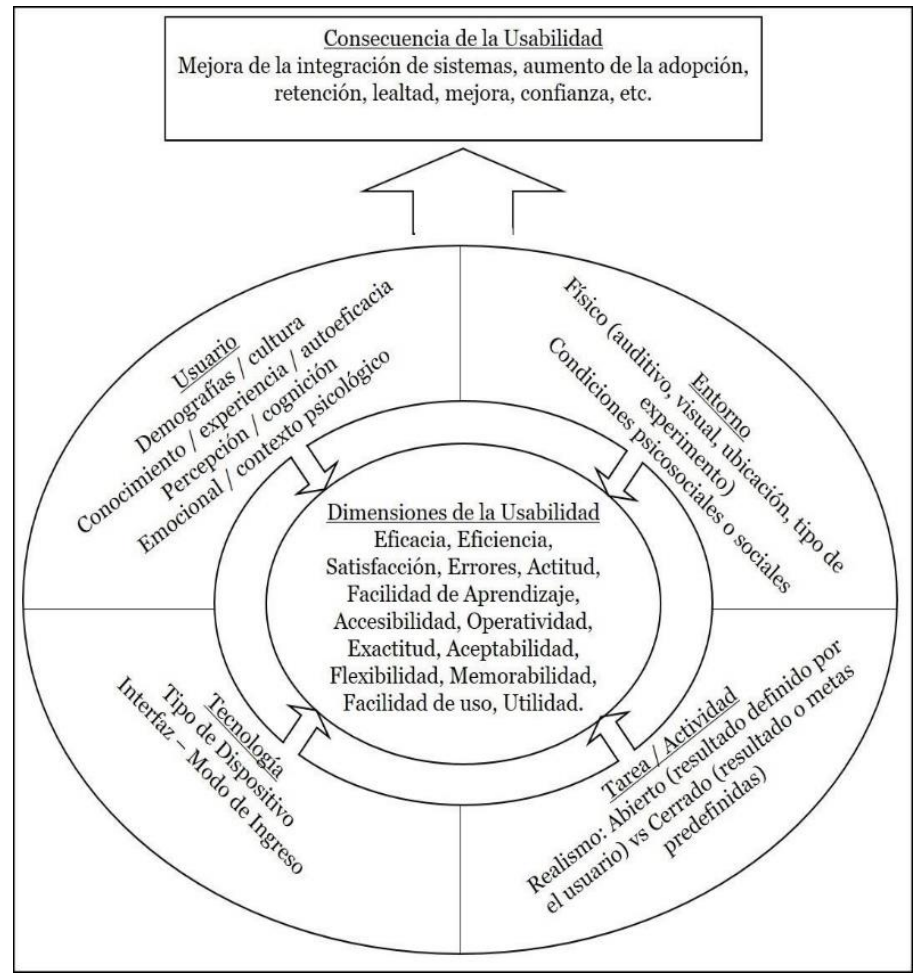




\section{Configuración informática}

El aplicativo Móvil fue desarrollado para dispositivos que dispongan del sistema Operativo Android con la versión mínima 4.0, haciendo que la aplicación se ejecute en casi el 99\% de dispositivos móviles Android. El desarrollo de la aplicación se realizó a través del IDE de desarrollo Android Studio con su respectivo SDK, haciendo uso del lenguaje de programación JAVA.

\section{Figura 5}

Arquitectura lógica de la aplicación.

\begin{tabular}{|c|c|}
\hline \multicolumn{2}{|c|}{ Interfaz Principal } \\
\hline \multicolumn{3}{|c|}{ IDE Android Studio } \\
\hline \multicolumn{3}{|c|}{ XML (Maquetación) } \\
\hline \multicolumn{2}{|c|}{ (Montenido Multimedia (Audios e } \\
Imágenes)
\end{tabular}

Publicación de la App en el Play Store

Al término del desarrollo de la aplicación se optó por publicarla de forma gratuita en la plataforma de distribución digital de aplicaciones móviles con sistema operativo Android, Play Store, y se le puede encontrar con el nombre WARMA en su página web oficial de la Universidad de Ayacucho Federico Froebel.

\section{RESULTADOS}

Este trabajo permitió comprobar la utilidad y pertinencia del uso del aplicativo móvil en los procesos de enseñanza del contenido básico de las asignaturas de matemáticas y lenguaje, en los niveles inicial y primario, en las instituciones educativas de las comunidades quechua hablantes de Ayacucho. Previó a los experimentos en la IE, se realizó un proceso de validación que consistió en las pruebas de performance, contenidos, usabilidad, este proceso permitió validar y afinar el aplicativo móvil.

En ambos niveles educativos se establecieron 2 grupos, el primer grupo recibió el aplicativo móvil como instrumento de enseñanza, además contaron individualmente con un orientador, el segundo grupo recibió clases con el método tradicional, que consistió en el uso de material impreso y también un orientador (en ambos casos no necesariamente el orientador fue el docente del curso). Participaron 06 niños en el nivel inicial ( 3 mujeres y 3 varones) y 10 niños del nivel primario (10 varones). De ellos más del $90 \%$ ya conocía y utiliza frecuentemente Smartphone (de sus padres). 


\section{Tabla 4}

Porcentaje De Aprendizaje En Alumnos

\begin{tabular}{lllllll}
\hline \multirow{2}{*}{ CAMUNIDADES } & \multicolumn{2}{c}{ Quinua } & \multicolumn{2}{c}{ Acosvinchos } & \multicolumn{2}{c}{ Chuschi } \\
\cline { 2 - 7 } & Sin la & Con la & Sin la & Con la & Sin la & Con la \\
& App & App & App & App & App & App \\
\hline Alfabeto & $75 \%$ & $100 \%$ & $75 \%$ & $100 \%$ & $75 \%$ & $87.5 \%$ \\
Los valores & $75 \%$ & $100 \%$ & $75 \%$ & $100 \%$ & $75 \%$ & $87.5 \%$ \\
La familia & $75 \%$ & $100 \%$ & $75 \%$ & $100 \%$ & $87.5 \%$ & $100 \%$ \\
El cuerpo Humano & $75 \%$ & $100 \%$ & $75 \%$ & $75 \%$ & $87.5 \%$ & $87.5 \%$ \\
Los sentidos del cuerpo & $50 \%$ & $100 \%$ & $75 \%$ & $100 \%$ & $75 \%$ & $87.5 \%$ \\
Los animales & $50 \%$ & $100 \%$ & $75 \%$ & $100 \%$ & $87.5 \%$ & $100 \%$ \\
Las cosas & $75 \%$ & $100 \%$ & $75 \%$ & $75 \%$ & $87.5 \%$ & $100 \%$ \\
Expresiones & $75 \%$ & $100 \%$ & $75 \%$ & $75 \%$ & $62.5 \%$ & $62.5 \%$ \\
Los números & $75 \%$ & $100 \%$ & $25 \%$ & $75 \%$ & $87.5 \%$ & $100 \%$ \\
Números ordinales & $75 \%$ & $100 \%$ & $50 \%$ & $100 \%$ & $75 \%$ & $87.5 \%$ \\
Figuras geométricas & $75 \%$ & $100 \%$ & $75 \%$ & $100 \%$ & $75 \%$ & $87.5 \%$ \\
Signos Aritméticos & $75 \%$ & $100 \%$ & $50 \%$ & $75 \%$ & $62.5 \%$ & $75 \%$ \\
Colores & $75 \%$ & $100 \%$ & $75 \%$ & $75 \%$ & $75 \%$ & $87.5 \%$ \\
\hline
\end{tabular}

Este evento consistió en 3 jornadas en las comunidades quechua hablantes de Ayacucho. Una vez concluida las enseñanzas se realizó la evaluación del aprendizaje. Previo a ello en el inicio se hizo una breve evaluación de los conocimientos y capacidades de los niños participantes, la mayoría conocía muy poco o nada los elementos básicos o contenidos de las asignaturas en la lengua quechua.

En ambos casos, el uso del aplicativo móvil como instrumento para la enseñanza, logró mejores resultados que la enseñanza tradicional, ello a partir de los datos obtenidos, donde los datos estadísticos de los estudiantes con resultados satisfactorios o que alcanzaron mejora es superior a los que usaron el método tradicional. Así mismo, es pertinente comentar el surgimiento de un fuerte interés, motivación y la aceptación de más del $80 \%$ de los niños de ambos sexos por el uso de la tecnología celular, otro factor clave que también generó interés y aceptación lo constituye los sonidos de la aplicación móvil, realizadas a partir de las voces de miembros de propia comunidad (familias y vecinos).

Los niños aprendieron con mayor facilidad los contenidos básicos de las signaturas de Matemática y Lenguaje, entre las que se encuentran el alfabeto (vocales y consonantes), los colores, los números, los animales, encontrando mayores dificultades en las frases comunes, la familia y partes del cuerpo. Finalmente, la experiencia de campo permitió analizar como el App Móvil puede complementar o apoyar al método tradicional o actual, teniendo en cuenta los principales problemas y limitaciones que afrontan actualmente las IE indígenas.

Con la publicación de la Aplicación Warma en la plataforma Play Store se logró difundir y masificar esta herramienta, es así que a un mes de su lanzamiento y publicación ya dispone de más de 2000 descargas, siendo el Perú el país que más descargas realizó, seguido por Brasil, Bolivia, Argentina y Chile. 


\section{Tabla 5}

Análisis Del Uso De La Aplicación Móvil

\begin{tabular}{|c|c|c|c|}
\hline $\mathbf{N}^{\circ}$ & Problemática & Con el método tradicional & Con la app móvil \\
\hline 1. & $\begin{array}{l}\text { Limitado } \\
\text { número de } \\
\text { docentes en las } \\
\text { IE de las } \\
\text { Comunidade } \\
\text { s. }\end{array}$ & $\begin{array}{l}\text { El número reducido de } \\
\text { docentes limita la calidad del } \\
\text { servicio educativo en las } \\
\text { comunidades indígenas. }\end{array}$ & $\begin{array}{l}\text { El App móvil facilita la enseñanza e } \\
\text { incluso autoaprendizaje de los } \\
\text { niños, pudiendo convertirse en un } \\
\text { complemento apropiado para } \\
\text { fortalecer el servicio educativo en } \\
\text { las comunidades indígenas }\end{array}$ \\
\hline 2. & $\begin{array}{l}\text { Docentes con } \\
\text { desconocimi } \\
\text { ento de la } \\
\text { lengua y cultura } \\
\text { local. }\end{array}$ & $\begin{array}{l}\text { Gran parte de los docentes } \\
\text { asignados a las IE indígenas, } \\
\text { no hablan la lengua ni cultura } \\
\text { local. Esto a la vez genera que } \\
\text { se desnaturalice la educación } \\
\text { bilingüe. }\end{array}$ & $\begin{array}{l}\text { El contenido interactivo está } \\
\text { disponible en la lengua local (menús } \\
\text { y contenidos temáticos). Esto } \\
\text { apoya incluso a docentes que no } \\
\text { hablan la lengua local }\end{array}$ \\
\hline 3. & $\begin{array}{l}\text { Material } \\
\text { Educativo } \\
\text { Suficiente }\end{array}$ & $\begin{array}{l}\text { La cantidad de textos y } \\
\text { materiales educativos en }\end{array}$ & $\begin{array}{l}\text { Por ser un recurso digital, el } \\
\text { contenido puede replicarse de } \\
\text { forma infinita. }\end{array}$ \\
\hline & & $\begin{array}{l}\text { las IE es muy limitado y } \\
\text { muchas veces inexistente }\end{array}$ & \\
\hline 4. & $\begin{array}{l}\text { Accesibilidad al } \\
\text { contenido } \\
\text { educativo. }\end{array}$ & $\begin{array}{l}\text { Las dificultades logísticas y } \\
\text { presupuestarias limitan la } \\
\text { distribución de contenido } \\
\text { apropiado en la lengua local } \\
\text { (llegar a las comunidades } \\
\text { indígenas es costoso) }\end{array}$ & $\begin{array}{l}\text { El App Móvil es accesible y } \\
\text { descargable fácilmente por internet } \\
\text { (aunque se requiere de un } \\
\text { dispositivo móvil, actualmente más } \\
\text { frecuente en las comunidades } \\
\text { indígenas). }\end{array}$ \\
\hline 5. & $\begin{array}{l}\text { Disponibilida } \\
\text { d en la lengua } \\
\text { nativa }\end{array}$ & $\begin{array}{l}\text { Pocos libros y textos en Perú } \\
\text { están disponibles en lenguas } \\
\text { indígenas. }\end{array}$ & $\begin{array}{l}\text { El App Móvil, está diseñado para } \\
\text { facilitar contenido educativo en } \\
\text { lenguas indígenas. }\end{array}$ \\
\hline
\end{tabular}

\section{REFERENCIAS BIBLIOGRÁFICAS}

El Comercio, (2018). Lenguas originarias del Perú: qué son, cuántas hay y otros datos que debes saber. Publicado el 25 de mayo. (04-03-19) https://elcomercio.pe/peru/lenguasoriginarias-peru-son-hay-cosas-debesnoticia-522310

El quechua, (s/f). La lengua mayoritaria entre las lengua indigenas. Publicado s/f. (0704-19) http://bvirtual.proeibandes.org/bvirtual/inge/10.pdf

Espinosa, O. (2017). Educación superiorpara indígenas de la Amazonía peruana: balance y desafíos. Recuperado de http://www.scielo.org.pe/pdf/anthro/v35n39/a05v35n39.pdf

El Universo, (2018). Intercultural. Publicado el 14 de abril. (05-04-19) https://cutt.ly/ZHsnTvK

Foros Ecuador, (2019). 14 Lenguas Originarias y Ancestrales del Ecuador - Idiomas indigenas ecuatorianos. Publicado el 18 de febrero. (04-04-19) https://cutt.ly/qHsnUsU

Instituto Socioambiental, (2028). Lenguas. Publicado el 15 de febrero. (03-04-19) https://pib.socioambiental.org/es/Lenguas

López, L. E., Küper, W. (2000). La educación intercultural bilingüe en América Latina: balance y perspectivas. Publicado en febrero $2000 . \quad$ (06-04-19) http://www.schwartzman.org.br/simon/delphi/pdf/lopes_comp1.pdf 
Minedu, (2013). Documento nacional de lenguas originarias del Perú. Lima, Perú. Corporación gráfica Navarrete.

Minedu, (2016). Resolución Ministerial. Publicado el 14 de diciembre. (07-04-19) https://cutt.ly/VHsnO7P

Moreno, G. (2017). Día internacional de la lengua materna. Publicado 21 de febrero. (040419) https:// cutt.ly/NHsnP3V

Nacional, (2019). Pueblos originarios. Publicado el 21 de febrero. (06-04-19) https://cutt.ly/DHsnSyy

National Geographic, (2018). Lenguas en peligro de extinción. Publicado el 16 de noviembre. (07-04-19) https:// cutt.ly/jHsnDtH

Rengifo, V. M. (2016). Impulso a la educación intercultural bilingüe de fortalecimiento y revitalización cultural y lingüistica. Publicado el 18 de junio. (06-04-19) https://cutt.ly/2HsnDBJ

UDAFF, (2019). Warma. https:// cutt.ly/pHsnFDu

UNESCO, (2019). ¿Por qué un año de las lenguas indígenas? Publicado, 2019. (04-0419) https://cutt.ly/JHsnGp0

UNESCO, (2019). UNESCO presenta el mapa de lenguas indigenas de Perú en peligro de extinción. Publicado el 21 de febrero. (05-04-19) https:/ / cutt.ly/DHsnGC9

UNESCO, (2019). Presentación del Año Internacional de las Lenguas Indigenas 2019. Publicado el 25 de enero. (07-04-19) https://cutt.ly/GHsnZaR

UMSA, (2018). Treinta de las treinta y seis lenguas nativas en Bolivia están en peligro de extinción. Publicado el 16 de octubre. (03-04-19) https:// cutt.ly/IHsnZ2i

Vida, (2018). Lenguas indígenas en México. Publicado el 21 de febrero. (06-04-19) https://www.mexico.mx/es/articles/lenguas-indigenas-mexico

\section{CITAR COMO:}

Gutiérrez Gómez, E., Ocampo Yahuarcani, I., Jeri Lagos, K. D., \& Saravia Llaja, L. A. (2019). Warma: aplicativo móvil para el aprendizaje de matemáticas y lenguaje en instituciones educativas de las comunidades quechua hablantes en Ayacucho. Puriq, 1(01), 68-82. https://doi.org/10.37073/puriq.1.01.57 